\title{
高价硫盐参与的多组分还原偶联反应：多官能团含砜分子的构建
}

\author{
刘冬梅天胜*
}

(中国科学院上海有机化学研究所 上海 200032)

\section{Multicomponent Reductive Cross-Coupling Involved by High-Valent Sulfur Salts: Straightforward Construction of Diversely Functionalized Sulfones}

\author{
Liu, Dong Mei, Tian-Sheng* \\ (Shanghai Institute of Organic Chemistry, Chinese Academy of Sciences, Shanghai 200032)
}

砜是构成小分子药物的重要结构单元. 因其在现代 药物分子中能平衡和控制药物的速率代谢和生物转 化 ${ }^{[1]}$, 所以一直位于有机化学研究的前沿, 并在商业药 物中得到了实际应用. 目前, 该类结构的合成通常用硫 醚在强氧化条件下来完成, 其强氧化性条件使得大部分 底物的官能团兼容性变差. 因此, 发展一种绿色、温和、 高原子经济性的方法是迫切的.

过渡金属催化的还原偶联反应是一种高效构筑碳碳键、碳一杂键的重要方法, 近年来, 无需预先形成有机 金属试剂的还原偶联反应得到了广泛的关注和发展. 其 中包括上海大学龚和贵 ${ }^{[2]}$ 和威斯康星大学麦迪逊分校 Daniel J. Weix ${ }^{[3]}$ 、巴黎综合理工大学 Corinne Gosmini 等 ${ }^{[4]}$ 都有出色的报道. 基于这一理论, 华东师范大学化 学与分子工程学院姜雪峰团队以高价硫盐焦亚硫酸钠 为 “ $\mathrm{SO}_{2}$ ” 源, 二价钯催化条件下实现了卤代芳烃和卤 代烷烃的还原插砜偶联反应, 可以实现高兼容性的含砜
类分子的构建(图 1) $)^{[5]}$.

首先，反应选取对甲基碘苯(1a)、2-溴-2-甲基丙烷 (2a)、焦亚硫酸钠为模板底物, 通过一系列的条件优化, 发现在 $10 \% \mathrm{PdCl}_{2}$ (dppf)催化下, 以 $20 \% \mathrm{dppf}$ 为配体，锡 作为还原剂, $\mathrm{K}_{2} \mathrm{HPO}_{4} \cdot 3 \mathrm{H}_{2} \mathrm{O}$ 为碱, 以 $96 \%$ 分离收率得到 目标产物. 随后，他们拓展了不同的卤代芳基以及烷基 源. 对于富电子和缺电子的碘苯, 反应都可以达到中等 偏上的产率; 而且邻位、间位取代, 吲哚、吡啶、噻吩 类的碘苯也不受影响. 在烷基源方面, 很多官能团比如 烯基(3ae、3af)、炔烃(3ag)等都可以兼容; 更值得关注 的是，反应还可以实现分子内还原偶联构建多构象、高 成环能系列中大 $(5 \sim 12$ 元 $)$ 环砜类结构, 其中七元环 (3at)给出了结构的单晶(图 2).

为了更进一步证实反应的适用性和实用性，他们还 实现了复杂分子之间的偶联，如糖、甾体、氨基酸和肽 之间的相互连接均可以用无机高价态硫作为连接子来

Our strategy

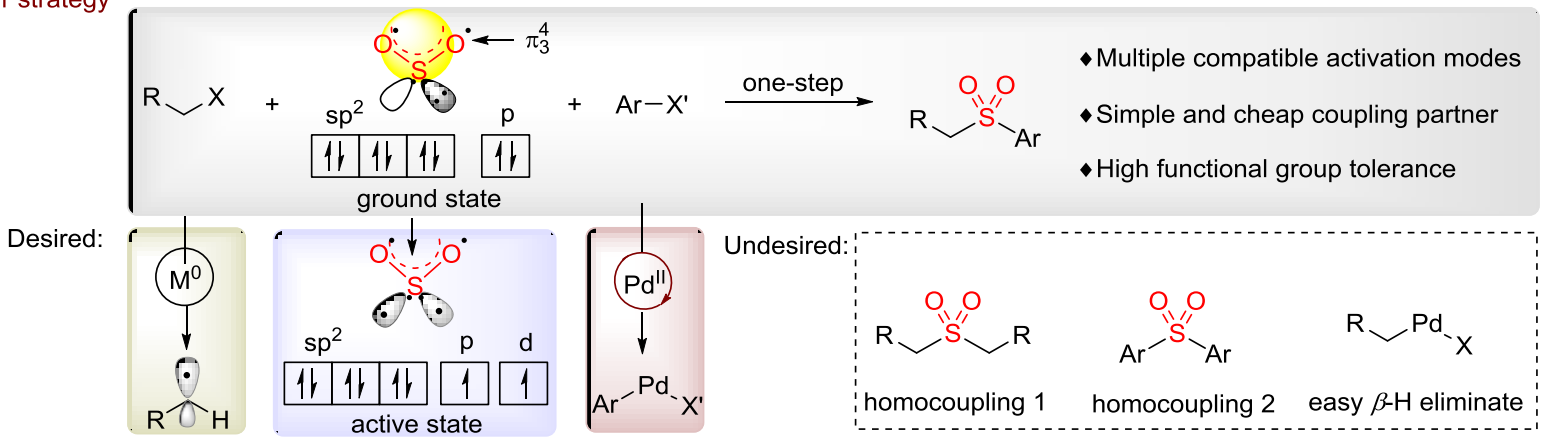

图 1 反应理论的提出

Figure 1 Proposal the reaction theory

* Corresponding author. E-mail: mei7900@ sioc.ac.cn. published online November 22, 2019. 


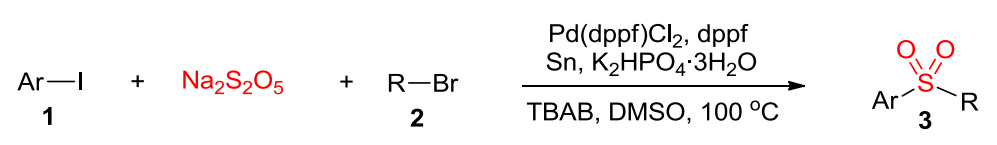

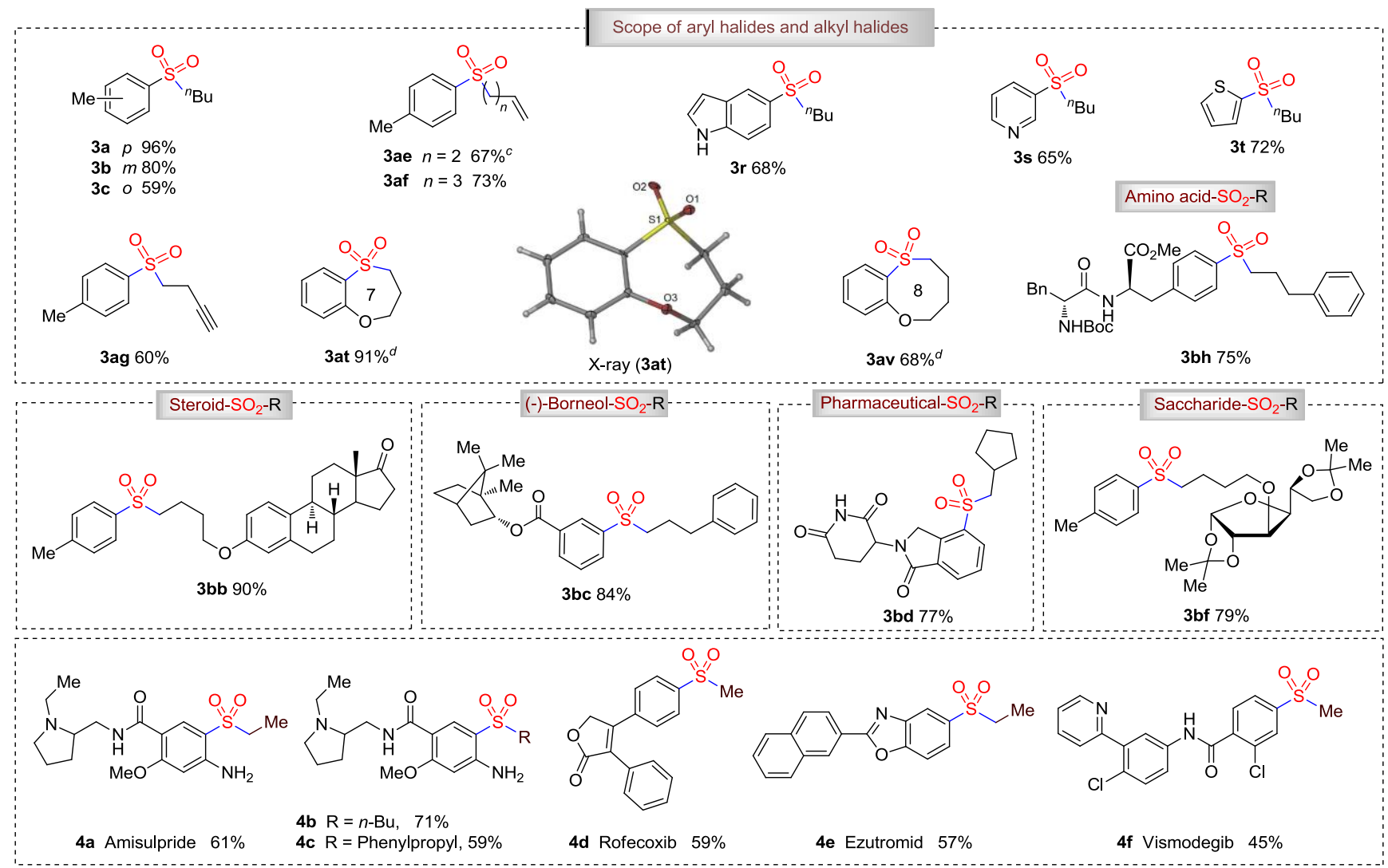

图 2 部分底物拓展

Figure 2 Part of the substrate

实现. 该研究还可以高效、高兼容地用于药物分子的构 建, 如抑制剂罗非昔布(Rofecoxib)、小分子抗肌萎缩蛋 白调节剂依柱曲米(Ezutromid) 以及罗氏开发的第一个 广谱细胞癌变药物维莫德吉(Vismodegib)等(图 2). 此方 法简单高效, 为含砜类药物的快速开发建立了一种新的 方案.

在反应机理验证方面, 他们在反应中加入四甲基哌 啶氧化物(TEMPO), 只能观察到微量的产物; 并且采用 自由基钟实验, 可以拿到 $38 \%$ 自由基开环的产物. 进一 步说明了反应中存在自由基; 在动力学同位素效应实验 中, 产物生成的速率对卤代芳基为 1.0 级动力学效应, 对烷基卤素为 0.188 级, 说明反应的决速步是零价钯对 卤代芳基的氧化加成, 而产生的烷基自由基过程是很快 的.

综合以上的实验结果, 他们提出了反应的可能机 理: (1)自由基偶联: 烷基溴代物通过 $\mathrm{Sn}$ 的单电子转移释 放出烷基自由, $\mathrm{Na}_{2} \mathrm{~S}_{2} \mathrm{O}_{5}$ 在溶液中是很好的 $\mathrm{SO}_{2}$ 源(具有 双自由基特征), 随后形成烷基砜自由基, 进一步被 $\mathrm{Sn}$
还原成砜负离子, 参与 $\mathrm{Pd}$ 催化循环. (2) Pd 催化循环: 二价钯在体系中被还原成零价钯，与芳基碘发生氧化加 成得到芳基钯物种，随后配体交换，还原消除得到芳基 烷基砜产物.

最后, 姜雪峰课题组巧妙地在卤代芳烃与烷烃的还 原偶联反应基础上, 引入砜基, 实现了高兼容性多敏感 官能化的含砜类分子的构建; 对于复杂药物分子间和分 子内, 都可以实现建砜精确、自由、高效, 为发展一种 绿色、广谱砜类化合物的方法提供了重要的科学和应用 价值.

\section{References}

[1] Ilardi, E. A.; Vitaku, E.; Njardarson, J. T. J. Med. Chem. 2014, 57, 2832.

[2] Wang, X.; Dai, Y.; Gong, H. Top. Curr. Chem. 2016, 374, 43.

[3] Weix, D. J. Acc. Chem. Res. 2015, 48, 1767.

[4] Knappke, C. E. I.; Grupe, S.; Gärtner, D.; Corpet, M.; Gosmini, C.; Wangelin, A. J. V. Chem. Eur. J. 2014, 20, 6828.

[5] Meng, Y.; Wang, M.; Jiang, X. Angew. Chem., Int. Ed. 2020, 59, 1346.

(Cheng, F.) 